\section{a Heighten Science \\ P U B L I C A T I O N S Corporation \\ ISSN \\ 2573-7724}

\title{
Time to Terminate LNT: Radiation Regulators Should Adopt LT
}

\author{
Jeffry A Siegel ${ }^{1 *}$, Bill Sacks² and James S Welsh ${ }^{3}$ \\ ${ }^{1}$ Nuclear Physics Enterprises, Marlton, NJ, USA \\ 2US FDA Medical Officer and Clinical Radiologist (Retired), Green Valley, AZ, USA \\ ${ }^{3}$ Dept of Radiation Oncology, Stritch School of Medicine Loyola University- Chicago, Maywood, \\ IL, USA
}

*Address for Correspondence: Jeffry A Siegel, President and CEO Nuclear Physics Enterprises, 4 Wedgewood Drive, Marlton, NJ, USA, Tel: (856) 899-9767; Email: nukephysics@comcast.net

Submitted: 29 May 2017

Approved: 23 June 2017

Published: 26 June 2017

Copyright: $\circledast 2017$ Siegel JA, et al. This is an open access article distributed under the Creative Commons Attribution License, which permits unrestricted use, distribution, and reproduction in any medium, provided the original work is properly cited.

Keywords: Linear No-Threshold (LNT) model; ALARA; Low-dose radiation risk; Adaptive response; Radiation protection; Radiation carcinogenesis

\section{ABSTRACT}

The linear no-threshold hypothesis (LNT)-the basis of radiation regulatory policy-extrapolates from observed high-dose harm to assumed low-dose harm, entailing that all ionizing radiation is harmful, by denying any biological response to damage and asserting cumulative lifetime harm, regardless of dose or dose rate. All aspects of LNT are demonstrably false. There are evolved biological responses that repair or remove radiogenic damage from low doses and dose rates, thereby averting acute harm and precluding the alleged cumulative damage. LNT and its offspring, the "as low as reasonably achievable" principle, do not err on the side of caution; neither is truly conservative. The public needs protection from radiophobia, rather than from low-dose radiation exposure. Neither radiation regulations nor medical practice should be based on LNT, but rather, at least as a first step, on a linear (down to a) threshold (LT) model.

\section{INTRODUCTION}

The linear no-threshold hypothesis (LNT) has been the scientific basis for virtually all world-wide radiation regulations and public policies for more than 70 years. Long assumed to be "conservative," policies based on LNT have recently been adversely affecting medical practice and evacuation procedures. However, LNT lacks valid scientific foundation. It is merely an assertion masquerading as scientific model $[1,2]$.

Still, even admitting invalidity as a possibility, many radiation protection and medical imaging professionals believe that LNT fosters "prudent" and "conservative" regulations that protect the public. In diagnostic radiology, LNT manifests itself in the "as low as reasonably achievable" (ALARA) principle, as well as in campaigns such as "Image Gently" (for children) and "Image Wisely" (for adults) that all call for lowering radiation dose, primarily for CT scans. Such calls inadvertently reinforce radiophobia, as the focus is solely on putative risks while implicitly denying its harmlessness or even benefit [2].

However, outside medical imaging, governmentally-imposed reactions to nuclear plant accidents decidedly refute assertions that lack of a threshold (i.e., the NT part of LNT) is protective to the population $[3,4]$. Thousands of unnecessary deaths have resulted from LNT-based forced evacuations at Fukushima and Chernobyl [5-8].

Epidemiological studies that appear to support LNT have neglected the biology, chemistry, and physics that should be the source and/or final arbiter of hypotheses concerning radiation hazards. Assertions that yield mathematically simple and convenient relationships should not be the basis for governmental policies [4]. 


\section{PETITIONS TO NRC AND RECOMMENDATIONS FROM ACMUI}

Three petitions were submitted to the United States Nuclear Regulatory Commission (NRC) on 23 June 2015 ( 80 FR 35870), requesting that use of LNT cease in favor of a model based on radiation-induced benefit at low doses (radiation hormesis). In its Final Report dated October 28, 2015, the NRC's Advisory Committee on the Medical Uses of Isotopes (ACMUI) advised the Commission (emphasis added):

The "correct" dose-response model for radiation carcinogenesis remains an unsettled scientific question. There is a large, and growing, body of scientific literature as well as mechanistic considerations which suggest that 1) the LNT model may overstate the carcinogenic risk of radiation at diagnostic medical, occupational, and environmental doses and 2) such low doses may, in fact, exert a hormetic (i.e., a beneficial or protective) effect. However, in the absence of definitive refutation of the LNT model and while strongly encouraging continued investigation critically comparing alternative models, regulatory authorities should exercise prudent (though not excessive) conservatism in formulating radiation protection standards. The ACMUI therefore recommends that, for the time being and subject to reconsideration as additional scientific evidence becomes available, the NRC continue to base the formulation of radiation protection standards on the LNT model.

Thus, the ACMUI acknowledged evidence for hormesis, yet recommended continued reliance on LNT for the indefinite future. Their advice wrongly assumes that LNT will provide protective policies pending "definitive refutation." In fact, hundreds of thousands of lives have been devastated or lost by the LNT-based overestimation of risk. Furthermore, the radiophobia inherent in applying ALARA (addressed in the petitions but unaddressed by ACMUI) diverts radiologists' and patients' attention from actual non-radiogenic risks, such as misdiagnoses from suboptimal images and/ or patient/parent avoidance of X-ray imaging. Although, not the aim of diagnostic imaging, ALARA also deprives patients of proven health benefits from the radiation itself. $^{2}$ Indeed, as with vitamin D synthesis, we all require a certain minimum ionizing radiation exposure (whether received from daily natural background radiation or the occasional low-dose medical imaging procedure) for optimal health $[9,10]$. ALARA is not merely useless, therefore, but actually harmful.

While demanding "definitive" refutation of LNT before policy is revised, the ACMUI leaves unspecified just what would constitute such refutation. They further fail to suggest either what might replace LNT or the requirement for its universal acceptance. The scientific "mainstream," to which proponents of LNT usually defer, are represented by advisory committees including NCRP, BEIR, and ICRP. Acknowledgement of refutation and acceptance of a replacement model by these organizations will likely be at best slow in coming, as their overlapping memberships have long endorsed, and enjoy reputations long invested in, LNT.

Interestingly, there is no demand for "definitive" evidence supporting LNT. Nor can any be found (or it already would have been cited by its avid proponents and universally accepted). In contrast, there are countless scientists who have already definitively refuted LNT, by either performing or acknowledging voluminous experimental and observational studies [1-3]. Thus, the ACMUI misassigns the burden of proof. This burden should be borne by those without data that validly support their advocacy of LNT, not by those with the preponderance of evidence refuting it.

\section{THE INTERNALLY CONTRADICTORY APPROACH OF LNT PROPO- NENTS}

Forced by opponents to finally take into account biological responses, LNT proponents have resorted to two attempts to salvage LNT: first, they erroneously claim that protective adaptive responses to low doses only partially offset the carcinogenic 
damage; second, they obfuscate by admitting that epidemiological studies cannot detect low-dose risks because of the noisy background, but they simultaneously deny without foundation the possibility of detecting harmlessness or even benefit [11]. Followers of the second course, however, reverse themselves when it serves their purposes. Many assert that large epidemiological studies, such as those by the International Agency for Research on Cancer (IARC), successfully demonstrate that chronic low-dose-rate radiation increases cancer mortality proportionally to cumulative dose.

For example, Dr. Little [12], of the National Cancer Institute took from the IARC studies that the mortality risk is increased by a mere $0.1 \%$ over a baseline total risk of some $25 \%$. Thus, after agreeing with those who claim that epidemiological studies cannot provide evidence for LNT because of statistical noise, he subsequently accepts uncritically the appearance of success, and specifically claims that $0.1 \%$ can even be detected against the rather large background noise. Thereby two mutually incompatible positions are sustained. But, as has been revealed, ${ }^{3}$ the illusion of IARC's success rests on circular reasoning, misassignment of exposure levels, and illegitimate statistical maneuvers-thereby resolving the apparent paradox though not absolving LNT proponents of the logical contradiction.

Credible evidence of carcinogenic risk at low acute doses ( $<100 \mathrm{mGy})$, such as those associated with medical imaging, is nonexistent; it is a hypothetical prediction derived from the demonstrably false LNT. Epidemiological studies at such low doses fail to validate LNT not because of statistical undetectability, but because the cancer risk is nonexistent, or perhaps even negative (hormetic) for the vast majority of peopleas hundreds of studies have demonstrated with statistical significance. That vast literature is either dismissed, if even cited, or ignored by those determined to preserve LNT as the basis of policy [13-15]. Science and the public thereby become their victims, even if unintended [4].

\section{REGULATORY AGENCIES SHOULD REJECT LNT/ALARA AND ADOPT A LINEAR THRESHOLD (LT) MODEL}

LNT, as we have reported [16], derives from incomplete, early-20th-century experimental measurements. Hermann Muller, in his 1946 Nobel Lecture, asserted unequivocally, in a stunning non-sequitur, that there is no threshold for harm all the way down to zero dose (or dose rate), despite the fact that his data only extended from very high doses down to a still high 4,000 mGy and from very high dose rates down to a still high $0.1 \mathrm{mGy} / \mathrm{min}$. Data gathered by Muller's colleagues within the following 2-3 years actually contradicted LNT, although they failed to recognize its significance. Their experimental evidence contained an unrecognized dose threshold, supporting a "linear (down to a threshold) threshold" (LT) model, and further, contained an unrecognized dose rate dependency, also with a threshold (at least when measured near their experimental dose threshold) [16].

Neither then nor since has any valid evidence supported the absence of a threshold (LNT) or the carcinogenicity of genuinely low-dose/low-dose-rate radiation. On the contrary, countless experimental and observational studies have shown that such doses do not cause cancer, but ironically help prevent it [13-15].

At low radiation exposures, initial radiation-induced damage is generally repaired or eliminated by the body's adaptive responses, including DNA repair (on the molecular level), apoptosis and bystander/rescue effects (on the cellular level), antioxidant production (on the tissue level), and immunological removal of any surviving damaged cells (on the organismal level). For example, it is known that CT exposure induces DNA double-strand breaks (DSBs) in patients, observable minutes after exposure. But repair of DSBs has been shown to occur subsequently [17]. In this study, in 22 out of 23 patients, the DSBs were repaired to initial (pre-CT) background levels within 24 hours. 
However, a decade later, studies in pediatric patients obtained data only at 5 minutes [18] or 1 hour [18], after the CT scans, and failed to permit time for repair of the observed and expected initial damage. The authors of the latter study even cite the earlier work of Löbrich et al. [17] and note that repair is possible at later intervals post CT. Asserting that further observations in children would have been "inappropriate," a "burden," and "too difficult," they merely grant that their "results are very preliminary and advise caution on over-drawing definitive, wide-reaching conclusions." However, ignoring their own advice, they conclude, "The results of our pilot study support the linear-no-threshold hypothesis $[14,15]$ at very low doses in young children. Our data suggest that even very low ionizing radiation exposure relevant to diagnostic CT exposure can leave a mark in the somatic DNA." Both these assertions are false and wholly unjustified by their truncated approach. The authors further conclude, again without valid justification that based on this (admittedly incomplete) evidence "When possible, CT exams should be limited or avoided by possibly applying non-ionizing radiation exposure techniques such as US or MRI." This is the kind of incomplete investigation that leads to unwarranted radiophobia and consequent refusal of many patients and physicians to utilize CT examinations when they are best suited for diagnoses needed to treat accurately and effectively.

Significantly, the Life Span Study (LSS) of Japanese atomic-bomb survivors, widely regarded as the single most important dataset for estimating radiation effects in humans from acute (high dose-rate) exposures, has recently been shown to suggest at the very least an LT, if not a hormetic, dose-response relationship [1,2].

Regulatory policy and medical practice should therefore be based on a linear (down to a) threshold (LT) model, below which there is either no risk or an actual health benefit for the vast majority of people. Implementing an LT model-a decision that could save countless lives-would obviate the radiophobic ALARA policy.

\section{CONCLUSION: ELIMINATE LNT/ALARA AND ADOPT LT}

Radiation regulation and medical imaging remain firmly wedded to the LNT fiction and ALARA. But neither LNT nor ALARA errs on the side of caution; rather both err strongly on the side of harm. The public deserves protection from radiophobiagenerating non-science, rather than from low-dose/dose-rate radiation. Regulatory and medical adherence to LNT and ALARA must therefore end and, as a first step, be replaced by LT.

\section{REFERENCES}

1. Siegel JA, Welsh JS. Does imaging technology cause cancer? Debunking the linear no-threshold model of radiation carcinogenesis. Technol Cancer Res Treat. 2016; 15: 249-256. Ref.: https://goo.gl/HXiFzN

2. Siegel JA, Pennington CW, Sacks B. Subjecting radiological imaging to the linear nothreshold hypothesis: a non sequitur of non-trivial proportion. J Nucl Med. 2017; 58: 1-6. Ref.: https://goo.gl/UEzLJ2

3. Sacks B, Meyerson G, Siegel JA. Epidemiology without biology: false paradigms, unfounded assumptions, and specious statistics in radiation science (with commentaries by Inge SchmitzFeuerhake and Christopher Busby and a reply by the authors). Biol Theory. 2016; 11: 69-101. Ref.: https://goo.gl/JyntJf

4. Siegel JA, Sacks B, Stabin MG. LNT 999. Health Physics News. 2015; 23-24.

5. The Japan Times. Fukushima stress deaths top 3/11 toll. 2014.

6. United Nations and International Atomic Energy Agency. Chernobyl Forum: Chernobyl's Legacy: Health, Environmental and Socio-Economic Impacts and Recommendations to the Governments of Belarus. The Russian Federation and Ukraine. 2006; 2003-2005. Ref.: https://goo.gl/Jcyz8H

7. Bennett B, Repacholi M, Carr Z. World Health Organization (WHO). Health Effects of the Chernobyl Accident and Special Care Programmes. Report of the UN Chernobyl Forum Expert Group "Health." Ed: WHO Press, World Health Organization, Geneva, Switzerland. 2006. 
8. United Nations Information Service. No Immediate Health Risks from Fukushima Nuclear Accident Says UN Expert Science Panel. 2013. Ref.: https://goo.gl/iKgKZc

9. Siegel JA, Marcus CS, Welsh JS, Pennington CW, Stabin MG. Regulatory application of the LNT hypothesis and ALARA to protect radiosensitive people is misguided. Health Phys News. 2016; 2324.

10. Mitchel RE. The dose window for radiation-induced protective adaptive responses. Dose-Response 2009; 8:192-208. Ref.: https://goo.gl/sroLFz

11. Little MP, Wakeford R, Tawn EJ, Bouffler SD, de Gonzalez AB. Risks associated with low doses and low dose rates of ionizing radiation: why linearity may be (almost) the best we can do. Radiology. 2009; 251: 6-12. Ref.: https://goo.gl/Xft1nN

12. Little MP. Ionising radiation in the workplace. BMJ. 2015; 351. Ref.: https://goo.gl/426YRP

13. Luckey TD. Radiation Hormesis. Boca Raton. 1991. Ref.: https://goo.gl/kHAxtT

14. Aurengo A, Averbeck D, Bonnin A. Dose-effect Relationships and Estimation of the Carcinogenic Effects of Low Doses of Ionizing Radiation. Paris: Académie des Sciences-Académie nationale de Médecine; 2005.

15. Feinendegen LE, Pollycove M, Neumann RD. Low-dose cancer risk modeling must recognize upregulation of protection. Dose-Response. 2009; 8: 227-252. Ref.: https://goo.gl/HUWd6v

16. Siegel JA, Pennington CW, Sacks B, Welsh JS. The birth of the illegitimate linear no-threshold model: an invalid paradigm for estimating risk following low-dose radiation exposure. Am J Clin Oncol. 2015. Ref.: https://goo.gl/5LgGTD

17. Löbrich M, Rief N, Kühne M, Heckmann M, Fleckenstein J, et al. In vivo formation and repair of DNA double strand breaks after computed tomography examinations. Proc Natl Acad Sci USA. 2005; 102: 8984-8989. Ref.: https://goo.gl/scZEvt

18. Vandevoorde C, Franck C, Bacher $\mathrm{K}$, Breysem L, Smet MH, et al. $\mathrm{Y}-\mathrm{H} 2 \mathrm{AX}$ foci as in vivo effect biomarker in children emphasize the importance to minimize X-ray doses in paediatric $\mathrm{CT}$ imaging. Eur Radiol. 2015; 25: 800-811. Ref.: https://goo.gl/Ny3MmR

19. Halm BM, Franke AA, Lai JF, Turner HC, Brenner DJ, et al. $y-H 2 A X$ foci are increased in lymphocytes in vivo in young children $1 \mathrm{~h}$ after very low-dose X-irradiation: a pilot study. Pediatr Radiol. 2014; 44: 1310-1317. Ref.: https://goo.gl/28NS2W 